\title{
Weekly Teriparatide Therapy Rapidly Accelerates Bone Healing in Pyogenic Spondylitis with Severe Osteoporosis
}

\author{
Akira Shinohara, Yutaka Ueno, Keishi Marumo \\ Department of Orthopaedic Surgery, Jikei University Kashiwa Hospital, Kashiwa, Japan
}

Pyogenic spondylitis is a frequently observed disease in orthopedics, and the number of cases is increasing. Some patients with pyogenic spondylitis suffer from vertebral destruction due to infection. The disease is typically treated with antibiotics, bed rest, spinal support, and lesion curettage; however, vigorous drug therapy against vertebral body destruction by pyogenic spondylitis has not been attempted. In this report, a case of pyogenic spondylitis with spinal destruction caused by infection and treated with once-weekly teriparatide administration is presented. Vertebral body erosion in cortical and cancellous areas by the infection was rapidly repaired after 6 weeks of once-weekly teriparatide treatment. Treatment with once-weekly teriparatide appears to be a new strategy for patients with severe osteoporosis suffering from pyogenic spondylitis.

Keywords: Bone density; Pyogenic spondylitis; Teriparatide; Percutaneous pedicle screws; Quality of life

\section{Introduction}

The number of patients who suffer from pyogenic spondylitis has been increasing; however, an effective treatment modality has yet to be established. Pyogenic spondylitis is commonly treated with antibiotics or bed rest in cases both with and without vertebral body destruction. Especially in patients with vertebral body destruction, the necessity of bed rest time increases, and patient's activities of daily living (ADL) and quality of life (QOL) decreases. For these reasons, the need for additional therapy has been recognized.

Teriparatide (PTH1-34) is a bone anabolic reagent that induces osteoblast activation, increases bone formation and bone mineral density (BMD) [1], and prevents vertebral fracture [2]. Moreover, it has been reported that teriparatide has an effect on fracture healing [3]. With these demonstrated clinical efficacies, teriparatide appears to have the potential to improve vertebral body destruction eroded by infection and improve both the ADL and QOL of patients.

The effects of once-weekly teriparatide in a patient with vertebral body destruction caused by pyogenic spondylitis are reported.

\section{Case Report}

A 78-year-old man presented with a fever of $39^{\circ} \mathrm{C}$, lumbar pain, and back pain. He had a history of type II diabetes complicated by hypertension, for which he had been taking $\alpha$-glucosidase inhibitors (voglibose, $0.2 \mathrm{mg} /$ day) and angiotensin II receptor blockers (candesartan

Received Aug 23, 2013; Revised Nov 6, 2013; Accepted Nov 13, 2013

Corresponding author: Akira Shinohara

Department of Orthopaedic Surgery, Jikei University Kashiwa Hospital,

163-1 Kashiwashita, Kashiwa, Chiba 277-8567, Japan

Tel: +81-4-7164-1111, Fax: +81-4-7163-3488, E-mail: shinohara75s1@gaea.ocn.ne.jp 
cilexetil, $2 \mathrm{mg}$ /day), respectively. Magnetic resonance imaging revealed changes in brightness of Th11, Th12, and L1. Plain radiographs (Fig. 1) and computed tomography (CT) (Fig. 2) revealed evidence of vertebral body destruction in Th12. Blood tests revealed both an increased Creactive protein level (CRP, $5.1 \mathrm{mg} / \mathrm{dL})$ and an increased white blood cell count (WBC, 7,900 cells $/ \mu \mathrm{L}$ ). Based on these findings, pyogenic spondylitis with vertebral body destruction was diagnosed. The patient also had severe osteoporosis, as indicated by a lumbar spine BMD T-score of -2.9 standard deviation (SD); however, the patient had not previously taken any osteoporosis medication.

The infection was treated with antibiotics (piperacillin and sultamicillin); the CRP level and WBC count re-

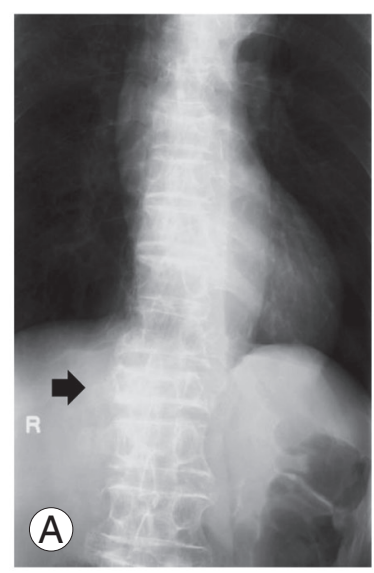

AP view

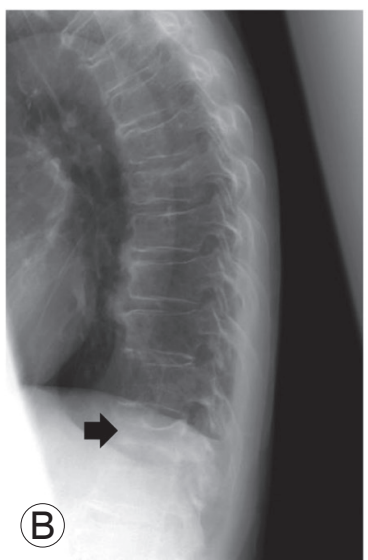

Lateral view

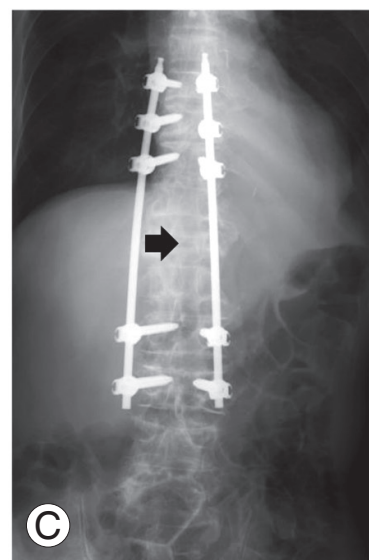

AP view

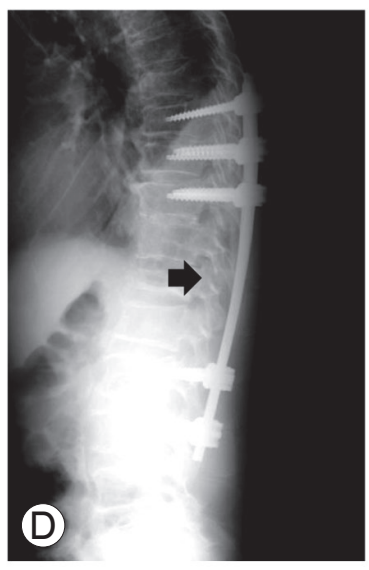

Lateral view

Before operation

After operation

Fig. 1. Plain radiographs at thoracic vertebra 12 (Th12) before and after operation. Plain radiographs show anteroposterior (AP) views $(\mathbf{A}, \mathbf{C})$ and lateral views $(\mathbf{B}, \mathbf{D})$ before and after operation. Arrows show Th12.

Before

administration
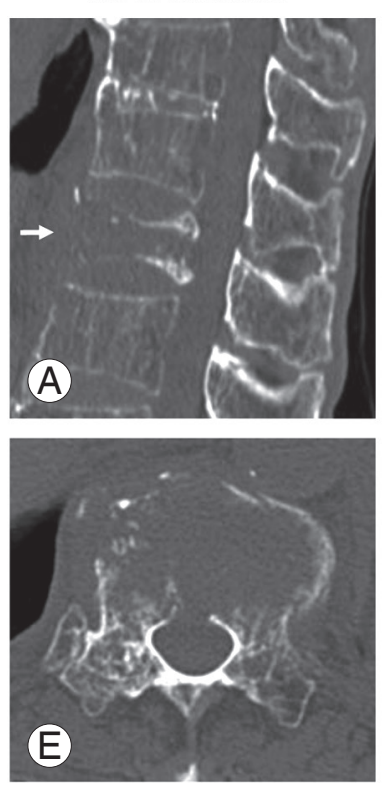

After 3 wk
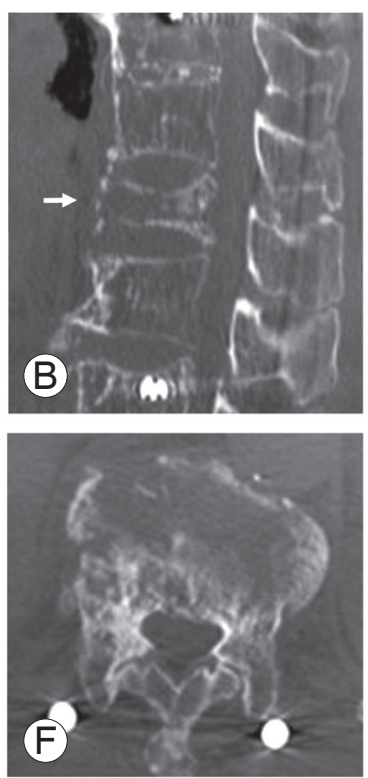

After 6 wk
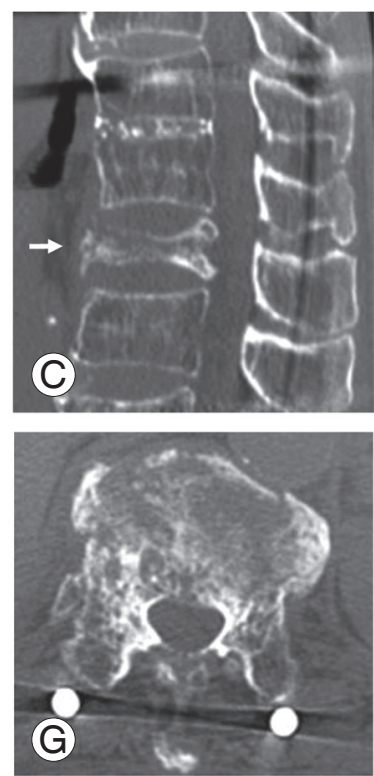

After 3 mo
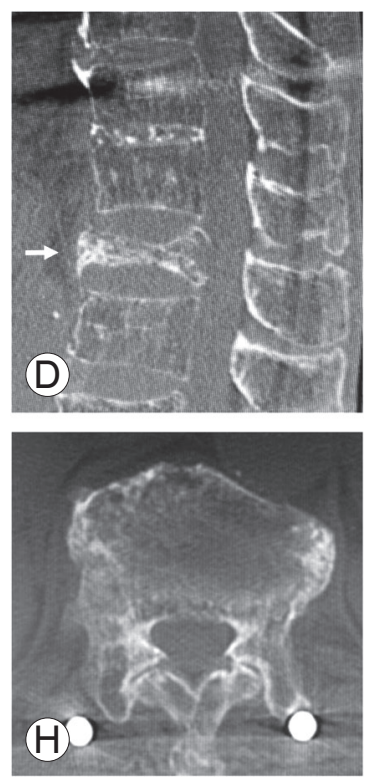

Fig. 2. Changes on computed tomography (CT) at thoracic vertebra 12 (Th12). CT images show sagittal (A-D) and coronal (E-H) sections before administration and 3 weeks, 6 weeks, and 3 months after administration of once-weekly teriparatide, respectively. Arrows show Th12. 
Table 1. Changes in BMD on dual energy $X$-ray absorptiometry

\begin{tabular}{lccc} 
Measurement site & Before administration & After 6 weeks & Change (\%) \\
Femoral neck & $-3.0 \mathrm{SD}$ & $-2.2 \mathrm{SD}$ & 17.6 \\
Total hip & $-3.6 \mathrm{SD}$ & $-3.3 \mathrm{SD}$ & 8.3 \\
\hline
\end{tabular}

BMD, bone mineral density; SD, standard deviation.

BMD is reported as the T-score.

turned to normal levels after eight weeks.

Along with antibiotic treatment, combined surgical and drug therapy for vertebral body destruction caused by the infection was performed. Minimally invasive percutaneous pedicle screw fixation was performed to alleviate the patient's back pain, and once-weekly, subcutaneous injections of teriparatide $(56.5 \mu \mathrm{g})$ were given to alleviate vertebral destruction and severe osteoporosis symptoms.

CT imaging was performed before and 3 weeks, 6 weeks, and 3 months after administration of weekly teriparatide (Fig. 1). Sagittal sections showed substantial bone formation over time relative to baseline in Th12. At week 6 of treatment, the Th12 endplate was more pronounced than at baseline. Coronal sections likewise showed substantial bone formation in and around Th12. Remarkably, cortical and cancellous bone in the vertebral body eroded by the infection showed rapid repair after 6 weeks of once-weekly teriparatide treatment (Fig. 2C, G). The bone formation efficacy of weekly teriparatide is also detected at 3 months (Fig. 2D, H).

Dual energy X-ray absorptiometry of the femoral neck and total hip was performed before and 6 weeks after administration of once-weekly teriparatide treatment (Table 1) in order to clarify the effect of the drug therapy on BMD. The femoral BMD increased to $17.6 \%$, and the total hip BMD increased to 8.3\% (Table 1).

Side effects of weekly teriparatide were shown in Teriparatide Once-Weekly Efficacy Research trial [2]: The common side effects were nausea, vomiting, headache, and abdominal discomfort. However, no side effects were observed in this patient.

\section{Discussion}

Spinal diseases are becoming more prevalent among the elderly, diabetics, and other immunocompromised patients. A growing number of these patients are also developing pyogenic spondylitis.
Antibiotic treatment and conservative treatment with external immobilization are first-line treatments for pyogenic spondylitis [4]. Surgical treatment should (sometimes) be considered in addition to conventional treatments either for patients resistant to conservative treatment, or for those who have severe bone destruction. Anterior curettage and bone grafting are first-line surgical treatments, and were recently shown to be beneficial when combined with posterior spinal fusion using spinal instrumentation [5]. The benefits are attributable to treatment that promptly suppresses the focus of infection, improves bone union, and stabilizes the spine, all of which allows patients to become ambulatory earlier. Mindful of these benefits, this case of pyogenic spondylitis was treated by performing posterior spinal fusion with percutaneous pedicle screws. Posterior spinal fusion with percutaneous pedicle screws features less bleeding and a shorter operating time than conventional fusion procedures; therefore, it is effective for patients with reduced resistance to disease (immunocompromised) or for those with poor overall health, as is often the case with pyogenic spondylitis patients.

The vertebral bodies of pyogenic spondylitis patients generally exhibit osteolytic change. Vertebral body stability must be maintained to allow bone regeneration. Although stabilizing vertebral bodies with posterior spinal fusion and spinal instrumentation as above, when possible, is helpful, the vertebral body osteolysis of the present patient made the cancellous bone of Th12 unrecognizable on CT scans. Therefore, conservative therapy alone would not have allowed the patient to become ambulatory, convincing us that treatment could take substantial time. Once-weekly teriparatide for his concomitant severe osteoporosis was attempted to determine whether it would promote bone formation at the infection site, in addition to treating his osteoporosis.

Teriparatide, which accelerates osteoblast-driven bone formation, increases BMD, and suppresses fractures [6], 
is used clinically worldwide to treat osteoporosis. Onceweekly subcutaneous teriparatide has been developed extensively in Japan and is now available in hospitals.

Surprisingly, once-weekly teriparatide resulted in bone formation after only 3 weeks of treatment. Cancellous and cortical bone clearly increased, thereby enhancing Th12 stability, after 6 weeks of treatment. We have never before observed bone formation this quickly in a patient undergoing posterior spinal fusion alone. The bone formation effect observed is thus attributable to once-weekly teriparatide.

Our experience with this patient prompted us to propose a new treatment regimen pairing teriparatide with existing treatment for pyogenic spondylitis. Teriparatide was recently shown to accelerate bone formation in osteonecrosis of the jaw $[7,8]$, the healing of fractures of the distal radius [3], pubis [9], atypical fractures [10,11], and bone union [12]. Our experience with this patient indicates that teriparatide is also highly effective in pyogenic spondylitis complicated by severe osteoporosis. The nearterm healing of bone destruction offered by teriparatide will surely improve patients' QOL.

\section{Conflict of Interest}

No potential conflict of interest relevant to this article was reported.

\section{References}

1. Reeve J, Hesp R, Williams D, et al. Anabolic effect of low doses of a fragment of human parathyroid hormone on the skeleton in postmenopausal osteoporosis. Lancet 1976;1:1035-8.

2. Nakamura T, Sugimoto T, Nakano T, et al. Randomized Teriparatide [human parathyroid hormone (PTH) 1-34] Once-Weekly Efficacy Research (TOWER) trial for examining the reduction in new vertebral fractures in subjects with primary osteoporosis and high fracture risk. J Clin Endocrinol Metab
2012;97:3097-106.

3. Aspenberg P, Johansson T. Teriparatide improves early callus formation in distal radial fractures. Acta Orthop 2010;81:234-6.

4. Cheung WY, Luk KD. Pyogenic spondylitis. Int Orthop 2012;36:397-404.

5. Saitou A, Suetsuna F, Mochizuki M. Surgical treatment using posterior instrumentation with pedicle screws for infectious spondylitis in thoraco-lumbar spine. Nihon Sekitsui Sekizuibyo Gakkai 2007;9:23-5.

6. Neer RM, Arnaud CD, Zanchetta JR, et al. Effect of parathyroid hormone (1-34) on fractures and bone mineral density in postmenopausal women with osteoporosis. N Engl J Med 2001;344:1434-41.

7. Lee JJ, Cheng SJ, Jeng JH, Chiang CP, Lau HP, Kok $\mathrm{SH}$. Successful treatment of advanced bisphosphonate-related osteonecrosis of the mandible with adjunctive teriparatide therapy. Head Neck 2011;33:1366-71.

8. Lau AN, Adachi JD. Resolution of osteonecrosis of the jaw after teriparatide [recombinant human PTH(1-34)] therapy. J Rheumatol 2009;36:1835-7.

9. Wu CC, Wei JC, Hsieh CP, Yu CT. Enhanced healing of sacral and pubic insufficiency fractures by teriparatide. J Rheumatol 2012;39:1306-7.

10. Carvalho NN, Voss LA, Almeida MO, Salgado CL, Bandeira F. Atypical femoral fractures during prolonged use of bisphosphonates: short-term responses to strontium ranelate and teriparatide. J Clin Endocrinol Metab 2011;96:2675-80.

11. Chiang CY, Zebaze RM, Ghasem-Zadeh A, IulianoBurns S, Hardidge A, Seeman E. Teriparatide improves bone quality and healing of atypical femoral fractures associated with bisphosphonate therapy. Bone 2013;52:360-5.

12. Mitani Y. Effective treatment of a steroid-induced femoral neck fracture nonunion with a once-weekly administration of teriparatide in a rheumatoid patient: a case report. Arch Osteoporos 2013;8:131. 